schiedenen Typen von Solen, die an verschiedenen Gefäßwänden entstanden sind.

9. Durch Reduktion von Silberoxyd mit Kohlenoxyd werden ebenfalls Metallsole erhalten, die jedoch unbeständiger sind.
Iо. Die Anwendung der kombinierten Analysenmethode auf Bredigs Silbersol hat ergeben, daß auch in diesem Oxyd vorhanden ist.

StraBburg, Dezember 1907.

(Eingegangen: Io. Januar.)

\title{
ZUR FRAGE VON DEN FALSCHEN GLEICHGEWICHTEN.
}

\section{Von E. H. Büchner.}

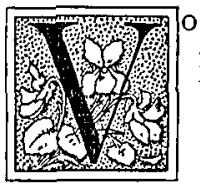

or mehreren Jahren hat Bodenstein in einer Reihe sehr sorgfältiger Experimentaluntersuchungen ${ }^{1}$ ) über die Bildung und Zersetzung von Selenwasserstoff, Schwefelwasserstoff und Wasserdampf zu zeigen versucht, daß die von $\mathrm{Duhem}$ und seinen Schülern angenommenen sogen. „falschen Gleichgewichtszustände" keine wirklichen Endzustände sind, sondern willkürlich herausgegriffene Punkte einer noch im Gange befindlichen Reaktion, welche nur so langsam fortschreitet, daß die Aenderungen innerhalb praktisch möglicher Versuchszeiten nicht merkbar sind. Wenn Bodenstein auch Resultate, welche von den von Pćlabon und Hélier gefundenen abwichen, erhielt, so hat seine Untersuchung doch nicht zur Entscheidung der Frage geführt.

Nun hat aber Duhem in seinem "Thermodynamique et Chimie" auf einen Fall hingewiesen 2), wo ein falsches Gleichgewicht im flüssigen Zustande auftritt, nämlich auf die von Engel entdeckte Reaktion:

$\mathrm{MgCO}_{3} \cdot 3 \mathrm{H}_{2} \mathrm{O}+\mathrm{KHCO}_{3}+n \mathrm{H}_{2} \mathrm{O}$ $\mathrm{MgCO}_{3} \cdot \mathrm{KHCO}_{3} \cdot \mathrm{H}_{2} \mathrm{O}+(n-\mathrm{I}) \mathrm{H}_{2} \mathrm{O}$.

Bringt man nämlich Magnesiumcarbonat mit einer Kaliumbicarbonatlosung zusammen, so wird ein wenig lösliches Doppelsalz von obenstehender Formel gebildet, wobei also der Kaliumgehalt der Lösung abnimmt. Uebergießt man aber anderseits das Doppelsalz mit Wasser, dann spaltet es sich in Magnesiumcarbonat, welches alsbald ausfällt, und Kaliumbicarbonat, das in Lösung bleibt. Und nun behauptet EngeI ${ }^{3}$ ), daß man auf dem einen oder anderen Wege - bei gleicher Temperatur ... nicht zu einer Lösung von derselben Zusammensetzung gelangt, obwohl man drei Komponenten und vier Phasen beisammen hat, welche bei bestimmter Temperatur, der Phasenregel gemäß, ein vollkommen bestimmtes System bilden müssen. Da nun in flüssigen Medien die störenden Einflüsse, welche bei Gasreaktionen fast immer wirksam zu sein scheinen, weniger oder gar nicht hervortreten, so schien mir in Anbetracht der Wichtigkeit der Frage eine Wiederholung der Versuche

I) Zeitschr. f. physik. Chemie 29 und 30 (1899).

2) S. 4I3.

3) Ann. Chim. Phys. [6], 7, 260.
Engels nicht überflüssig, um so mehr, als dieselben zu verschiedenen Bedenken Anlaß geben. Er gibt z. B. an, daß die Zeit, während welcher die Lösung sich nicht mehr änderte, öfters nur $r$ bis 3 Stunden betrug.

Obwohl Engel Versuche bei 15,30 und $40^{\circ}$ angestellt hat, habe ich mich auf die Temperaturen 20 und $30^{0}$ beschränkt, weil bei ungefähr ${ }_{1} 5^{\circ}$ ein anderes Hydrat $\left(\mathrm{MgCO}_{3} \cdot \mathrm{H}_{2} \mathrm{O}\right)$ auftritt, und oberhalb etwa $35^{\circ}$ Kaliumbicarbonatlösung schon merklich Kohlensäure abspalte.t, welche Erscheinung das Gleichgewicht beeinflussen wird.

Das $\mathrm{MgCO}_{3} \cdot 3 \mathrm{H}_{2} \mathrm{O}$ und das Doppelsalz wurden nach den Angaben von von Knorre ${ }^{1}$ ) dargestellt und deren Reinheit durch Analyse festgestellt. Es wurde dann eine größere Flasche mit einer genügenden Menge Magnesiumcarbonat beschickt, dieselbe ganz mit etwa $0,6 \mathrm{n}$. Bicarbonatlösung (welche aus vollkommen reinem $\mathrm{KHCO}_{3}$ bereitet war) angefüllt ${ }^{2}$ ), mit einem Gummistopfen verschlossen und in einen genau regulierten Ostwaldschen Thermostaten gehängt, wo dieselbe durch einen Motor in fortwährender Bewegung erhalten wurde. Daneben wurde gleichzeitig eine zweite Flasche hineingebracht, welche mit Doppelsalz und Wasser - oder $\mathrm{KHCO}_{3}$ - Lösung von kleinerem Gehalt als die Gleichgewichtskonzentration - ebenfalls vollständig angefüllt wurde. Nach bestimmten Zeiten wurde mittels einer Pipette mit vorgelegtem Watteröhrchen eine Menge Lösung herausgenommen, rasch mit kaltem Wasser verdünnt und mit Schwefelsäure titriert, mit Methylorange als Indikator, so daß man die Gesamtmenge an $M g^{*}+K^{\prime}$ - Ionen bestimmt. Die Resultate sind in folgender Tabelle vereinigt, in welcher neben der Zeit die Anzahl Kubikzentimeter $0,2 \mathrm{n}$. Schwefelsäure, welche nötig sind, um $10 \mathrm{ccm}$ der filtrierten Lösung $\mathrm{zu}$ neutralisieren, angegeben worden ist.

Die Tabelle weist, von kleinen Unregelmäßigkeiten abgesehen, unzweifelhaft aus, daß von

I) Zeitschr. f. anorg. Chemie 34, 260 (I903).

2) Die vollständige Füllung geschah mit der Absicht, eventuelle Kohlensäureabspaltung so viel wie möglich einzuschränken. Jetzt war sogar nach der letzten Probenahme nur ein verhältnismäßig kleiner Gasraum vorhanden. 
Temperatur $20^{\circ}$.

\begin{tabular}{|c|c|c|c|}
\hline \multirow{2}{*}{$\begin{array}{l}\text { Zeit } \\
\text { in } \\
\text { Tagen }\end{array}$} & \multicolumn{3}{|c|}{$\begin{array}{c}\text { Kubikzentimeter } \\
0,2 \mathrm{n} . \mathrm{H}_{2} \mathrm{SO}_{4}\end{array}$} \\
\hline & $\underset{\mathrm{I}}{\text { Spa }}$ & II & Bildung \\
\hline I & 9,9 & - & - \\
\hline 4 & I 7,7 & $\mathrm{I} 4,3$ & 26,8 \\
\hline 8 & I 7,9 & $\mathrm{I}_{6}, 4$ & 17.9 \\
\hline $\mathrm{I}_{5}$ & I 7,9 & 17,2 & - \\
\hline 25 & - & 17,8 & 17,7 \\
\hline $3^{2}$ & - & 18,0 & 17,8 \\
\hline
\end{tabular}

Temperatur $30^{\circ}$.

\begin{tabular}{|c|c|c|c|}
\hline \multirow{2}{*}{$\begin{array}{c}\text { Zeit } \\
\text { in } \\
\text { Tagen }\end{array}$} & \multicolumn{3}{|c|}{$\begin{array}{c}\text { Kubikzentimeter } \\
\text { o,2 n. } \mathrm{H}_{2} \mathrm{SO}_{4}\end{array}$} \\
\hline & $\underset{I}{\text { Sp }}$ & & Bildung. \\
\hline 0 & - & 24,8 & 28,75 \\
\hline I & 24,4 & - & - \\
\hline 4 & 一 & 25,2 & 25.4 \\
\hline 7 & 252 & - & 25.3 \\
\hline 12 & 25,5 & $25, \mathrm{I}$ & - \\
\hline 24 & - & 25, & 25.3 \\
\hline
\end{tabular}

beiden Seiten her derselbe Endzustand erreicht wird, und es also nur eine Loosung gibt, die mit den beiden Salzen im Gleichgewicht ist. Dieselbe liegt zwischen den beiden von Engel erhaltenen; dieser fand nämlich für $3^{\circ}$, in unsere Maße umgerechnet, in den Spaltungsversuchen $24,75 \mathrm{ccm}$, bei der Bildung dagegen $27,25 \mathrm{ccm}$. Es ist hiermit in einem Falle, in dem man die einzelnen experimentellen Bedingungen genau übersieht, bewiesen, daß das angebliche falsche Gleichgewicht nur durch ungenügende Genauigkeit der Versuche vorgetäuscht war; genau dasselbe Resultat also, wie Bodenstein erhielt, und durch welches er zum Ausspruch geführt wurde, da $\beta$ den falschen Gleichgewichten keine reale Existenz zukomme ${ }^{1}$ ). Daß aber dieser Schluß mir nicht vollkommen richtig gefaßt erscheint, möchte ich jetzt noch kurz begründen.

Die Versuche lehren uns unwidersprechlich, $\mathrm{daB}$ es in gewissen Systemen eine Reihe $\mathrm{Zu}$ stände gibt, welche mit der Zeit keine Aenderung erleiden - welche daher nach der Definition als Gleichgewichte zu betrachten sind - , welche aber von der Thermodynamik nicht vorhergesehen werden. Abgesehen von den Versuchen Pélabons und Héliers, brauche ich nur an

I) a. a. $\mathrm{O}, \mathrm{Bd} . \mathbf{3 0}, \mathrm{I} 37$. die absichtlich angestellten Experimente von Viktor Meyer und $\operatorname{Raum}^{1}$ ) zu erinnern, welche zeigten, daß bei der Temperatur von $100{ }^{0}$ sich aus Knallgas selbst in 218 Tagen keine Spur Wasser bildete. Diesen unleugbaren Widerspruch zwischen Theorie und Experiment kann man auf zwei Wegen zu lösen versuchen. Entweder man unterwirft die Theorie einer genauen Prufung und verbessert dieselbe, wenn man dazu Anlaß findet, oder man zweifelt an der Richtigkeit der Versuchsresultate. Duhem tut das erstere und findet wirklich im Gebäude der Thermodynamik einen Baustein, welcher ihm nicht tauglich scheint; indem er diesen auswirft und durch einen anderen ersetzt, entwickelt er in ganz richtiger Weise eine Theorie, welche mit den Versuchen in Uebereinstimmung ist. Dagegen folgt Bodenstein, wie bekannt, dem zweiten Weg; auch dieser führt, wie Duhem selbst bemerkt hat ${ }^{2}$ ), theoretisch zum Ziel. Experimentell wird man, nach den Resultaten von Bodensteins sehr genauen Versuchen, ebenfalls wohl zu keiner Entscheidung kommen können, und mir scheint es daher nur die Frage zu sein, welcher Weg der einfachere ist. Wenn ich dann schließlich im Sinne Bodensteins entscheide, also annehme, daß die Reaktion mit unmerklicher Geschwindigkeit fortschreitet, dann geschieht dies in erster Linie darum, weil seine Auffassung als eine einfache Extrapolation bekannter Tatsachen aus der Lehre von der Reaktionsgeschwindigkeit gelten kann, während die Duhemsche Theorie nur ad hoc entwickelt und sonst - wenigstens bis jetzt - nicht mit anderen Tatsachen in der Chemie in Verbindung gebracht worden ist.

Anorganisch-Chemisches Laboratorium der Universität zu Amsterdam.

(Eingegangen: 20. Januar.)

I) Berichte 28, 2804 (I895).

2) a. a. O., S. $4^{\circ 6 .}$

\section{PATENTLITERATUR.}

\section{AUSZÜGE AUS DEUTSCHEN PATENTSCHRIFTEN.}

A. Stanfield und L. B. Reynolds. Verfahren und Ofen $z$ um Verbüten von bleiischen Zinkerzen bezw. zinkischen Bleierzen durch Reduktion mit Kohle. D. R. P. 183470 vom 3.6.05. Es handelt sich um die Verhüttung solcher Erze, die für das gewöhnliche Bleiverfahren zu zinkreich oder für das Zinkverfahren zu bleireich sind, derart, daß in derselben Operation beide Metalle getrennt gewonnen werden. Das Verfahren beruht auf der elektrischen Erhitzung einer Schlacke, die leichter ist als Blei, so daß das Blei sich auf dem Boden sammelt, während das Zink in Form von Zinkrauch oberhalb der Schlacke bleibt und abgesogen wird. Fig. 24 zeigt einen dazu geeigneten Ofen, Fig. 25 stellt den Schnitt in $\operatorname{der}$ Linie $A A$ von Fig. 24, Fig. 26 den
Schnitt in der Linie $B B$ dar. Der Ofen setzt sich zusammen aus dem eigentlichen Schmelz- oder Reaktionsraum $k$ und den seitlich daran liegenden Kondensationsräumen für Zinkmetall $l l$; die Räume stehen durch Kanäle $m$ miteinander in Verbindung. Von $/$ aus führen Kanäle $o$ zu den Räumen $n$. Vor den Kanälen $o$ liegen Praliplatten $r$. Durch die Decke der Schmelzkammer $k$ ragen ein oder mehrere Beschickungsschächte $s$ so tief in die Kammer hinein, daß ihre Mündungen etwas höher als die Sohle der Kanäle $m$ liegen. Sie werden durch die Begichtungsvorrichtung $s^{1}$ mit dem Gut beschickt. Rechts und links von $s$ stehen Reihen von Elektroden $t$ aus Kohle, durch die Stopfbüchsen $v$ abgedichtet. Die Schmelzkammer enthält an einem Ende den Abstich 2 